\title{
REDUCING THE GAP BETWEEN WORK AS DONE AND WORK AS IMAGINED ON CONSTRUCTION SAFETY SUPPORTED BY UAS
}

\author{
Roseneia Melo ${ }^{1}$ \\ Dayana Costa ${ }^{2}$ \\ 1) PhD Student, Federal University of Bahia, Brazil \\ 2) Associate Professor, Federal University of Bahia, Brazil
}

\begin{abstract}
Safety management (SM) is considered a complex task during the construction phase, given the number of high-risk activities that can lead to accidents. The differences between work as done (WAD) and work as imagined (WAI) is one of the main barriers faced by safety management. The Resilience Engineering stand out that improving in safety performance cannot be simply achieved through the use of procedures and barriers, but through the continuous monitoring. On the other hand, the use of Unmanned Aerial Systems (UAS) technology can contribute to the safety management system to support the monitoring of the daily work. This study aims to evaluate how work is performed from RE perspective through the application of the safety checklist based on the assets collected with UASs on site, focusing on the cast-in-place concrete wall constructive process, once it involves high risk of accidents during construction. For this, an exploratory case study in a construction project was conducted in Brazil, involving the following steps: (a) development of a safety monitoring protocol using UASs, (b) field tests for monitoring safety conditions with UASs along 35 weeks, and (c) data analysis. As contribution, this work identifies potential improvements on safety procedures aiming to reduce the differences between prescribed and actual work. In addition, the UASs can be used to perform regular and redundant safety inspections providing information to support managers' decisionmaking.
\end{abstract}

Keywords: Safety Monitoring, Resilience Engineering, Unmanned Aerial Systems (UAS), Work as done (WAD), Prescribed and actual work, Construction sites.

\section{INTRODUCTION}

Construction projects can easily be characterized as complex systems, due to the high degree of interconnectivity and interdependence between components/process (Perrow, 1984; Saurin, 2017). Also, the construction sector is known as one of the most hazardous industries worldwide presenting high accident rates (Dias, 2009). Safety Management System (SMS) is responsible for the identification, assessment, control and evaluation of hazards that can lead to accidents. However, a large number of Job Hazard Areas (JHAs) are not identified because of the dynamism and complexity faced by the construction environment (Guo et al., 2017). Dias (2009) also argue that the efficiency and effectiveness of the existing systems for implementing and monitoring safety have been questioned.

(C) 2019 Melo \& Costa. This is an Open Access article distributed under the terms of the Creative Commons AttributionNonCommercial 4.0 International License (http://creativecommons.org/licenses/by-nc/4.0), permitting all non-commercial use, distribution, and reproduction in any medium, provided the original work is properly cited.

ISBN: 978-91-88898-41-8

DOI: https://doi.org/10.15626/rea8.02 
The main shortcomings faced by safety management are related to the inefficient safety training, the safety planning failures, and the ineffective control of working conditions, being the failure to identify safety hazards the major cause of accidents in construction sites (Guo et al., 2017). The lack of real information impedes effective risk assessment and monitoring of how work is performed. Therefore, those tasks end up being mainly based on construction designs (Guo et al. 2017). Other issue reported by Guo et al. (2017) and Saurin et al. (2005) is related to the monitoring process not having the appropriate technological support to make the risk assessment more realistic.

Therefore, despite the advances in construction management, the effectiveness of the traditional Safety view, also known as Safety-I, has been often questioned. The Safety-I approach aims to identify the causes and factors that trigger adverse events (accidents or incidents), eliminate their causes and / or improve the barriers (Hollnagel et al. 2015). However, this approach analyzes people, technology and work context independently, considering only causal relationships and neglecting the interactions between them (Clegg, 2000, Hollnagel et al., 2015). As a consequence, it influences safety performance, since the construction project deals with social and technical factors at different hierarchy levels. Resilience Engineering (RE), also known as Safety-II, brings a change of perspective that emphasizes the ability to succeed under unwanted conditions. RE seeks to understand how people under pressure deal with complex situations with high levels of uncertainty and variability (Hollnagel, 2006). This approach also pointed out the importance to understand the gap between work as done and work as imagined, for improving worker's awareness and skills to deal with unwanted events.

On the other hand, recent research has shown the use of emerging technologies, such as Unmanned Aerial Systems (UASs) to support safety management on construction sites (Irizarry and Costa, 2016; Gheisari and Esmaelli, 2016, Melo et al., 2017). Melo et al. (2017). Irizarry and Costa (2016) stand out UASs as potential assets to support the workplace monitoring of activities which involve high accident risk and difficulty of visualization, providing information for safety managers about real work conditions. Thus, the use of the UAS technology could help fill in the gaps regarding the lack of information during risk assessment and safety monitoring at construction sites.

Therefore, this study has as its motivation the use of Resilience Engineering and the UAS technology to better understand the differences between prescribed and actual work through the monitoring of workplace conditions. An exploratory case study was carried out in a construction project in Brazil focused on concrete wall constructive process, due to the high risk of accidents during execution and the growth in its adoption in the low-income housing projects. Also, the concrete wall process presents some characteristic pointed out by Perrow (1984) regarding complex systems, such as the high risk of accidents during execution influenced by the tight spacing of equipment, proximate productions steps, invariant sequence of production, substitution of suppliers limited and the possibility of little slack.

\section{BACKGROUND}

\subsection{Resilience Engineering (RE)}

The concept of 'resilience' has been discussed in different fields of knowledge. In Engineering, it can be understood as the ability of how people, alone or together, cope with everyday situations - large and small - by adjusting their performance to the conditions. An organisation's performance is resilient if it can function as required under expected and unexpected conditions alike (changes/disturbances/opportunities) (Hollnagel, 2018). 
Resilience is also related to the ability of organizations or systems to manage conflicts and adjust boundary conditions to better accommodate demands, anticipate and control threats, to mitigate the pressure on production goals and maintain an acceptable level of risk (Woods, 2006, Hale and Heijer, 2006). Thus, the RE seeks to explore and discuss the trade-offs between safety and production faced by organizations (Hale and Heijer, 2006, Woods, 2006).

Since failures or drift are related to the working conditions that exceed safe work boundaries (Rasmussen, 1997), some abilities and principles need to be developed to understand how the system adapts and how to manage and control events that may disturb the continuity of workflow. According to Dekker (2003) there are two ways to advance safety: (1) the application of the procedure as a sequence of rules, and (2) the application of the procedure as a cognitive activity. However, there are some situations in which the procedures are not applicable, highlighting the importance of the human capacity to judge when and how to adapt procedures for eventual circumstances (Dekker, 2003).

Schafer et al. (2008) point out that resilience cannot simply be integrated by using procedures, guidelines, personal protective equipment and barriers, but through the continuous monitoring of the system's performance. According to Nakajima et al. (2017) the WAI is important for plan and manage work, once should represent the best understanding of how work will be performed. Moreover, Hollnagel et al. (2015) pointed out that WAI, consist of an idealistic view, which ignores how individuals and organizations adjusting to current conditions. In the other hand, WAD represents the reality of everyday work and reflects performance variability that underlies how work is actually done (Nakajima et al., 2017; Saurin et al., 2017). How to capture WAD in a sociotechnical system still a challenge, once each system or organization has its own components which could interact in unexpected ways. As proof of that, there is no preestablished instruction how systems evolve.

Besides that, Rasmussen (1997) argues about the importance of making the boundaries explicit and known, by giving the workers opportunities to develop skills to cope with unsafe work boundaries. From this point of view, the organizations must monitor and understand the reasons behind the gap between prescribed and actual work and develop ways to support people's ability to adapt to the unexpected situations and reduce the incidence of violations and errors (Dekker, 2003, Costella et al. 2015). In practice, procedures are often adapted by workers considering the appraisal of the local situation, which not necessary imply that the violations must be directly be associated with accidents (Dekker, 2003, Costella et al., 2015).

Reason (1990) classified violations as routine and exceptional violations. The routine violations are associated the adaptations performed by workers during the executed the tasks by means which differ from prescribed procedures. Sometimes, the violation is view as a normal way into the daily practice that it is no longer identified as an illegal act. In some context, violations may be understood as an inevitable consequence to achieve the established performance in complex systems (Polet et al., 2003). Nevertheless, given the dynamic and complex nature of construction work, these kinds of studies have been sparsely investigated, pointing the need for studies to understand and evaluate the differences between prescribed and actual work at construction sites in order to meet safety rules (Borys, 2012).

\subsection{Unmanned Aerial Systems (UAS) applied to safety purpose in construction}

Unmanned Aerial Systems (UASs), also known as drones, are defined as aircrafts that function without any pilot onboard (Irizarry et al., 2012). Initially, UASs were used in military applications, but recently, the potential use of UAS for safety purposes has gained significant attention. Irizarry et al. (2012) carried out an exploratory analysis concerning the use of UASs to assist in safety inspection on construction sites. Despite the fact that safety monitoring was 
limited to inspecting the use of hardhats by construction workers, the results showed that the UASs can help in the safety inspection process and supporting the decision-making. Kim and Irizarry (2015) have identified benefits and critical factors that influence the UAS performance for safety inspection. Among the main benefits, Kim and Irizarry (2015) highlight the effective monitoring of the workplace, the easy identification of the problems and agility in the correction of potential dangers. Regarding critical factors, these authors emphasize characteristics related to project (location, size, and cost), staff experience with UASs and attitudes toward UAS implementation.

Gheisari and Esmaelli (2016) listed 16 risks situations that could be improved through the use of UASs, and 15 of these situations are related to the monitoring and inspection of construction activities and just one related to post-accident investigation. Melo et al. (2017) evaluated the applicability of UASs for safety inspection at construction projects. They developed and validated a safety checklist with the possible safety requirements to be inspected using UASs and two case studies were carried out. The results showed that the collected assets can collaborate with the inspection process through better visualization of the working conditions, particularly with the inspection of fall protection equipment.

Although studies pointed out the potential of UASs applied to safety, corroborating with the increased transparency of processes, improved ease of problem identification, agility in the decision-making process and shorter inspection time (Irizarry et al., 2015; Kim and Irizarry, 2015; Melo et al., 2017). New studies need to be developed aiming to monitor work as done, anticipate risks and unsafe situations not detected in design phase, increase worker awareness by training using visual resources (photos and videos), and promote proactive actions.

\section{METHOD}

The case study research strategy was adopted in this study and was performed according to the following stages: (a) literature review about RE and the use of UASs for safety purposes in construction, (b) development of a safety monitoring protocol using UASs, (c) field tests for safety conditions monitoring with UASs, and (d) the analysis of compliance based on the safety standards and analysis of the main differences between prescribed and actual work based on safety rules, with emphasis in the cast-in-place concrete wall process. These stages will be detailed as following.

The study was conducted in a residential project, known as Project A, from October 2017 to March 2018. The main features of the project and safety team are presented in Table 1.

\begin{tabular}{l|l}
\hline \multicolumn{1}{c|}{ Description of project } & \multicolumn{1}{c}{ Description of Safety Team } \\
\hline Residential low-income housing project & Company Safety Manager, Safety \\
Land Area: $22,800 \mathrm{~m}^{2}$ & Supervisor, Safety coordinator and two \\
Total of 400 units & Safety Personnel. \\
Construction time: 16 months & \\
Constructive processes: Concrete wall structure & \\
\hline
\end{tabular}

Table 1: Features of Case Study

\subsection{Development of safety monitoring protocol by UAS}

This step aimed to improve the safety monitoring protocol with UASs develop by Melo et al. (2017). The protocol involves the following steps:

(a) the UAS mission planning defines the point of interest and involves a check list for the UAS take-off and landing procedures (Irizarry et al., 2015; Melo et al. 2017), take into consideration 
the safety criteria established by the National Agency for Civil Aviation in Brazil (Melo et al., 2017).

(b) a manual flight is carried out for collection of the visual assets (photos and videos) with the UAS (DJI Phantom 3 Advanced) using a DJI app.

(c) the data collected during flight is stored and processed in a flight log data form and the visual asset data base.

(d) a checklist for UASs safety monitoring was developed based on the Project 1's own checklist for assessment of the construction site safety conditions (only the items that could be visualized using the UASs were considered). The checklist included 105 items, divided into 11 categories (organization and housekeeping, storage of materials, safety nets, earthwork and foundations, concrete pouring process, masonry process, concrete wall structure, roof, stairs and ramps, scaffolding and equipment operation). This work will give more emphasis in the monitoring of the concrete wall process since it is considered a process with high risk of accidents, a total of 15 safety items were monitored by checklist using UAS presented in Table 2 .

(e) the visual assets are analyzed based on the checklist which aims to identify compliance according to Brazilian Safety Regulation, called NR 18 - Working and Environmental Conditions in the Construction Industry (Brasil, 2015). In addition, an analysis of differences between prescribed and real work were carried out for cast-in-place concrete wall process based on the visual assets collected with UAS. For each monitoring, a report is produced and shared with the safety and production managers.

\begin{tabular}{|c|c|}
\hline Item & Description \\
\hline 1 & $\begin{array}{l}\text { Worker using fall arrest systems, connected to the lifeline independent of the } \\
\text { support structure }\end{array}$ \\
\hline 2 & $\begin{array}{l}\text { Peripheral protections (guardrails) and work platform are complete and properly } \\
\text { installed }\end{array}$ \\
\hline 3 & Work platforms with adequately closing (guardrails) at their ends. \\
\hline 4 & Workers use PPE permanently, according to the activity risks \\
\hline 5 & Workplace organized during the assembly and disassembly of panels \\
\hline 6 & Place of lifting load operation is isolated and signalized \\
\hline 7 & $\begin{array}{l}\text { Isolation throughout the workplace which involving assembly and disassembly of } \\
\text { panels, signaling the risk of falling materials or tools. }\end{array}$ \\
\hline 8 & There are caps on the ends of rebar \\
\hline 9 & $\begin{array}{l}\text { Remains of the cut outs of the screens being discarded in bays keeping the } \\
\text { organization and cleaning during the process. }\end{array}$ \\
\hline 10 & $\begin{array}{l}\text { Waste stored appropriately using gutters fixed to the structure for transport and } \\
\text { collection }\end{array}$ \\
\hline 11 & Scaffolding with guardrail and footer systems in the work structure \\
\hline 12 & Lifeline support is fixed in the structure through pins, angled wedges and ties. \\
\hline 13 & Hand tools tied to prevent accidental fall. \\
\hline 14 & $\begin{array}{l}\text { During the assembly and disassembly of the external scaffolding, the worker uses } \\
\text { fall arrest systems. }\end{array}$ \\
\hline 15 & $\begin{array}{l}\text { The safety cable is attached to the structure independent of the structure of the } \\
\text { scaffold. }\end{array}$ \\
\hline
\end{tabular}

Table 2: Items monitored in the Concrete Wall Process 


\subsection{Field test for safety monitoring using UAS}

This step aimed to perform tests for monitoring safety conditions on the jobsite and identify the safe work limits through the visual assets collected with UASs. A total of 35 flights were performed in Project 1 with an average time of 15 minutes each flight. The concrete wall process had 19 week-monitored. A total of 2,863 photos were taken and 75 minutes of video were recorded. After the flights, a feedback session with Safety Personnel from Project A for immediate assessment was performed. All visual assets collected during the monitoring as well as a report were available to the stakeholders to assist in their decision-making process.

During the execution of the project, two debriefing with the project top management were carried out to discuss the conflicts between safety and production, the non-conformities and the good practices identified in the monitoring.

\subsection{Data analysis}

The first analysis of this study aimed to identify the safety compliance based on the safety checklist and data collected with the UAS. The safety criteria were evaluated according to Safety Standards, highlighting unsafe conditions and safety/production conflicts visualized through the visual assets collected. The Safety Compliance Indicator (SCI) was calculated as the ratio of the total weights of items in conformity with the total weights of all items applied (including non-conformity items). The weights of the safety criteria vary according to the degree of risk (low, medium and high risk). This indicator was chosen because it allows analyzing how work is performance and they monitor either the presence of safety or actions that have been adopted to create safety (Saurin et al., 2017).

The second analysis aimed to evaluate the main differences between prescribed and actual work regarding to the execution of safety rules, focusing on the cast-in-place concrete wall constructive process. The identification of risk and control measures were carried out based on safety plans developed by company and legal documents, such as the Program of Prevention of Accident Risks (PPAR) and the Program of Work Conditions and Environment in the Construction Industry. However, despite the plans contain all risks associated the constructive method adopted, this study focused only on risk of fall from height and from falling objects which can be viewed through the assets collected with UAS.

\section{RESULTS AND DISCUSSIONS}

\subsection{Analysis of safety compliance with UAS with emphasis on concrete wall process}

Figure 1 presents results of the Safety Compliance Indicator. In general, the results show an improvement in safety conditions during the monitoring between October 2017 and August 2018, as presented in the tendency line (linear weekly). In the first two visits, the results were below $70 \%$, which is justified by the period of the temporary installation and jobsite layout implementation. From the seventh visit on, a high-compliance rate (above 90\%) is observed, representing the fulfilment of the safety standards requirements and the commitment to safety management.

Lower compliance results were observed during visits 22-24 and 30-31, resulting from failures in the cast-in-place concrete wall constructive process (guardrails systems were not totally installed ahead of the activity execution and workers were not using safety belt attached in lifeline systems). On visit 34, the compliance was impacted by the absence of Collective Protective Equipment (CPE), such as horizontal lifeline systems, during the roof installation.

Despite the high level of general safety compliance (Figure 1), a record of twelve accidents on the jobsite during this period were observed, one of them was characterized as a severe injury, 
the others were classified as minor injuries. The results prove that a high safety compliance rate does not necessarily imply an absence of accidents, since it is not possible to predict all events.

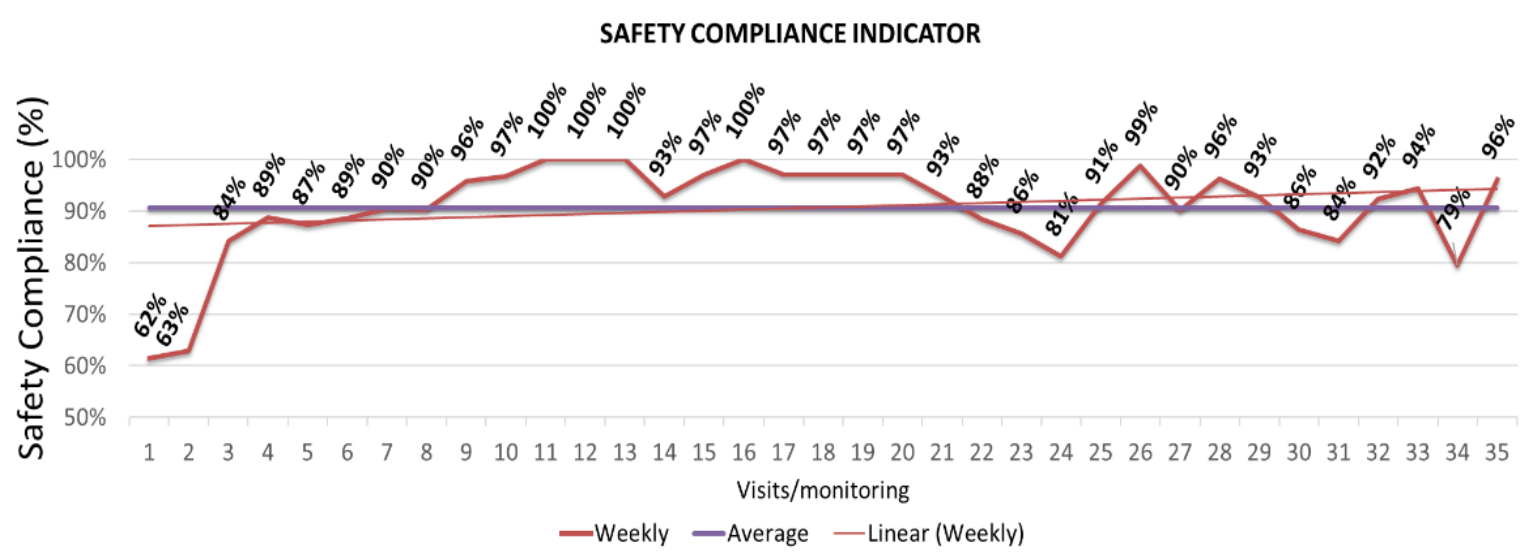

Figure 1 - Safety compliance indicator at construction site

A more detailed analysis of the non-conformities and deviations was carried out in order to identify the process that had most impact on the safety performance. A total of 102 nonconformities (35 items) was identified, highlighting the most impact processes, such as: the organization and cleaning of the construction site with $28 \%$ and the cast-in-place concrete wall system with $20 \%$. Thus, based on the results of analysis of the non-conformities and the safety team perceptions, the cast-in-place concrete wall process was identified as the critical process due to the difficulty of monitoring and its complexity.

The cast-in-place concrete wall system is made with ready-mix concrete placed into removable metallic forms erected on site for the execution of walls and slabs of reinforced concrete. This process includes the sub-processes of metallic panels assembly, rebar assembly, electrical and hydraulic installation, ready-mix concrete placed and metallic panels disassembly. In addition, during the process, the assembly and disassembly of collective protections, such as guardrails, safety platform and lifeline system and quality and safety inspections is also included.

Comparing the general safety compliance indicator with the safety compliance of cast-in-place concrete wall process (Figure 2), most of cast-in-place concrete wall process compliance results were below the overall performance of the project. These findings highlight the need to investigate the main differences between prescribed and actual work, to ensure the work safe boundary, especially in process with high risk of accidents.

\section{SAFETY COMPLIANCE INDICATOR}

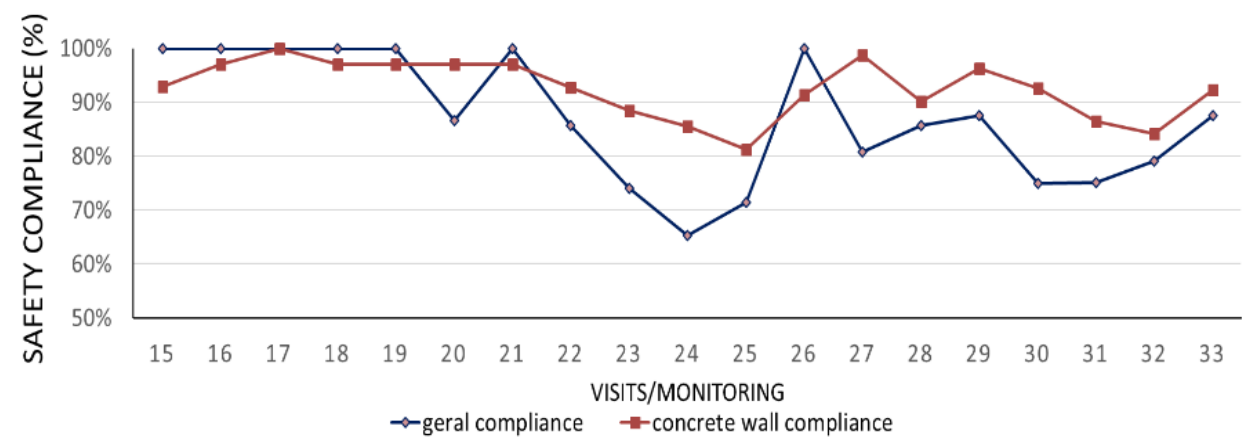

Figure 2-Safety compliance indicator for concrete wall process 


\subsection{Analysis of prescribed and actual work concerning cast-in-place concrete wall process}

The identification of potential risks was carried out in the design phase. This risk is monitored during the construction phase by using safety checklist, which items are shown in Table 2. According to safety monitoring, the safety checklist is applied monthly by the company, although the work operation needs to be inspected daily, because the daily production cycle adopted. Only a visual inspection is done by personal safety, in which none record is created about everyday safety performance. Other point for improvement that need to be stand out is that the risk regarding to the prohibition of work overlapping identified in risk assessment is not monitored using the safety checklist.

A more detailed analysis about the differences between prescribed and actual work, considering the items inspected (Table 2), is shown Figure 3. A total of 20 non-conformities regarding to the concrete process were identified and most of them (80\%) are related to: worker using fall arrest systems, connected to the lifeline independent of the support structure (item 1), peripheral protections (guardrails) and work platform are complete and properly installed (item 2) and work platforms with adequately closing at their ends (item 3). These three items presented are concerning to Collective Protection Equipment (CPE), which must be properly installed before the activity begin, which demonstrating fails in the executions of safety procedures.

\section{NON-CONFORMITIES OF CONCRETE WALL PROCESS}
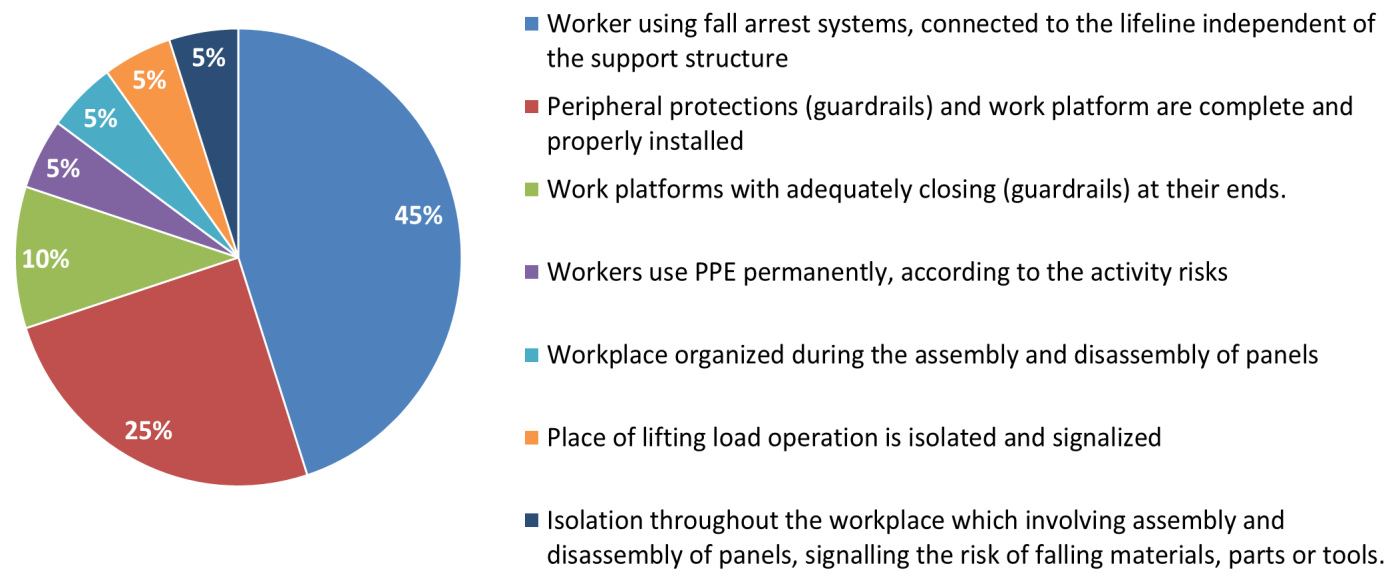

Figure 3-Non-compliances identified in the concrete wall process

Most of items classified as non-conformity can be associated with management factors (insufficient safety training, inefficient safety personnel supervision, outdated procedures, safety planning failures and lack of detailed in projects of CPE) and human factors (human behavior). Figure 4 presents some situations that exceed the safe work boundaries were recorded by the visual assets collected with UASs.

Among the hazardous situations identified, the following ones can be highlighted: (a) although the workers were using a safety belt, there were no lifeline systems on the slab to be trussed (Figure 6a); there was not peripheral protection) to ensure workers safety conditions during the steel assembly activity (Figure 6b); during the movement of metals panels, there were no lifeline systems on the slab to be trussed (Figure 6c); and during the process of removing suspended scaffold, the worker in the slab edge was not protected by fall protection systems (Figure 6d); besides that they were operating tasks at different elevations in the same direction, which must be prohibited increasing the risk of accident. 

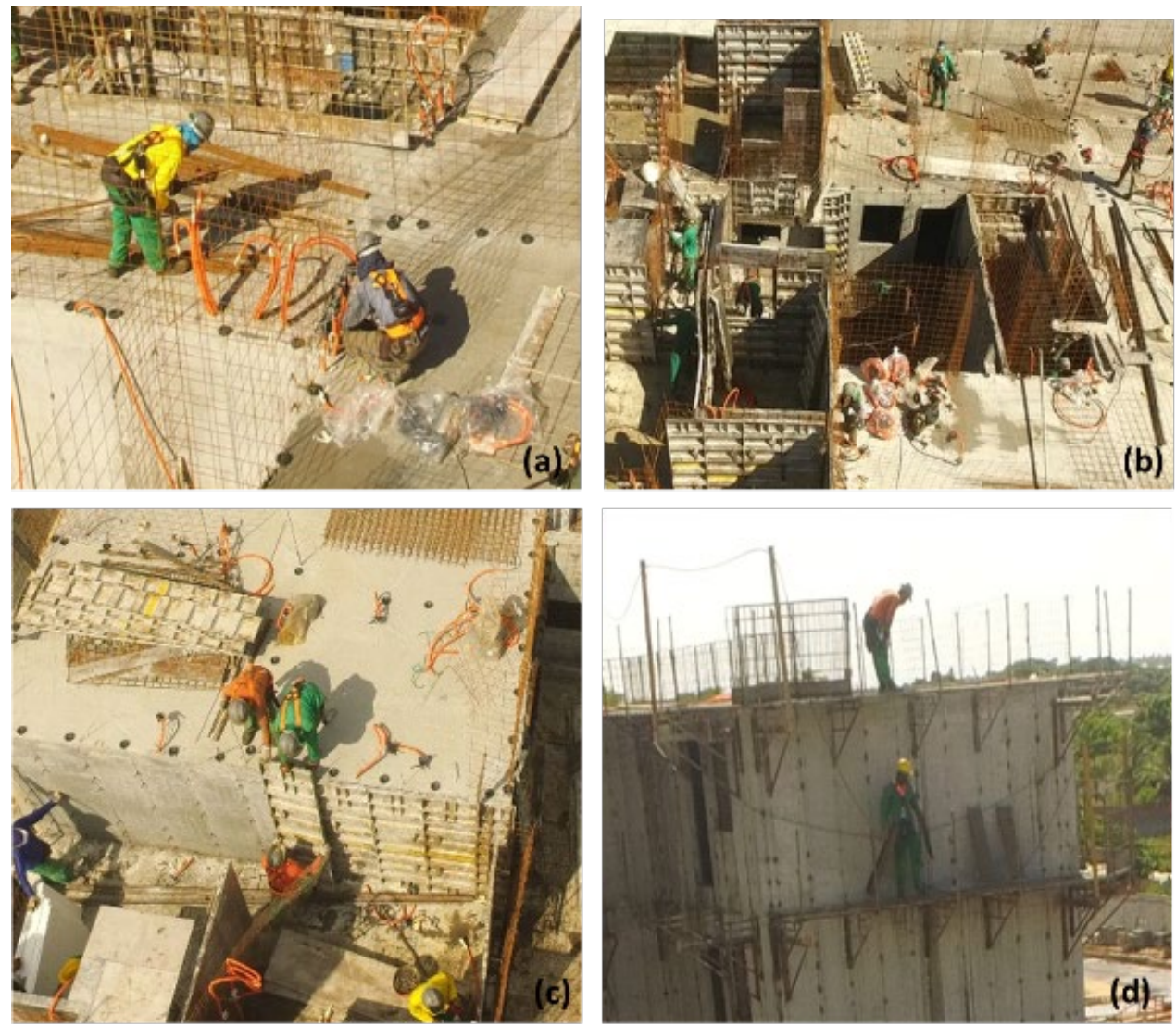

Figure 4-Non-compliance identified in the concrete wall process

\section{CONCLUSION}

The case study developed on a construction site aimed to evaluate the safety compliance with Brazilian safety standards through the application of the checklist based on the assets collected with UASs, and to investigate how the UASs can contribute to the safety management system to support the monitoring of the daily work. In this study, a safety compliance indicator was calculated and situations that exceeded the safe work boundaries were identified to propose improvements in a timely manner.

The results show that a high safety compliance does not necessarily imply an absence of accidents, since it is not possible to predict all events. Moreover, it is essential to develop workers' capacity for their adaptation and awareness of the unsafe work limits, as well as to understand the differences between the prescribed and actual work, according to Rasmussen (1997) and Costella et al. (2015). The analysis of prescribed and actual work shows that despite the risk assessment in the design phase and the preposition of control measures in the safety plans, in practice safety procedures are often put aside to achieve the production goals.

Some opportunities for improvement were observed. The use of technological resources (photos and videos) to analyze the actual work (work as done) can allow the identification of divergence aspects between practice and procedures, and also can be used as a resource to understand the safe work boundaries during safety training. Another opportunity is the need for integrating the safety and production planning to improve the safety working conditions, given the need to remove safety constraints ahead of scheduled activity execution. All of these opportunities seek to contribute to improve the working conditions, applying RE concepts. 
Moreover, this research contributes with the improvement of a UAS safety protocol to monitor workplace conditions, providing information for compliance analysis in timely manner and better understanding about the differences between prescribed and actual work.

The results shown that UASs can be used to perform regular and redundant safety inspections providing information to support managers' decision-making, especially for tasks which involve a high risk of accidents, promoting greater transparency. The visual assets collected with UASs can also be used for feedback about the safety plans and procedures and to increase the workers' awareness by means of safety training. As limitations, only external criteria regarding safety were monitored with UASs, and the data collected with UAS providing instantaneous results about a short space-time. New studies are under development to implement UAS technology to improve the safety management on construction projects and understand the reasons concerning the differences between prescribed and actual work.

\section{REFERENCES}

Borys, D. (2012). The role of safe work method statements in the Australian construction industry. Safety science 50, 210-220. doi:10.1016/j.ssci.2011.08.010

Clegg, C. W. (2000). Sociotechnical principles for system design. Applied ergonomics 3, 463-477. https://doi.org/10.1016/S0003-6870(00)00009-0

Costella, M. F., Saurin, T. A., Cambraia, F. B. and Bortolosso, H. (2015). Violation or resilience? A comparison between two frameworks for making sense of work-as-done. In: 6th Symposium on Resilience Engineering Association, Lisbon, Portugal.

Dekker, S. (2003). Failure to adapt or adaptations that fail: contrasting models on procedures and safety. Applied ergonomics 34, pp. 233-238. https://doi.org/10.1016/S0003-6870(03)00031-0

Dias, L. A. (2009). Inspecting occupational safety and health in the construction industry. International Training Centre of the International Labour Organization. Report ISBN 978-92-9049-489-8

Gheisari, M. and Esmaeili, B. (2016). Unmanned Aerial Systems (UAS) for construction safety applications. Proceedings of the Construction Research Congress, San Juan, pp. 2642-2650.

Guo, H., Yu, Y. and Skitmore, M. (2017). Visualization technology-based construction safety management: A review. Automation in Construction 73, 135-144. https://doi.org/10.1016/j.autcon.2016.10.004

Hale, A. and Heijer, T. (2006). Defining resilience. Resilience Engineering: concepts and precepts, pp. 35-40. Ashgate.

Hollnagel, E. (2006). Resilience - the Challenge of the Unstable. Resilience engineering: Concepts and precepts, pp. 9-17. Ashgate.

Hollnagel, E., Wears, R. L. and Braithwaite, J. (2015). From Safety-I to Safety-II: A white paper. The resilient health care net: published simultaneously by the University of Southern Denmark, University of Florida, USA, and Macquarie University, Australia.

Hollnagel, E. (2017). Safety-II in practice: developing the resilience potentials. Routledge.

Irizarry, J. and Costa, D.B. (2016). Exploratory Study of Potential Applications of Unmanned Aerial Systems for Construction Management Tasks. Journal of Management in Engineering 32.

Irizarry, J., Gheisari, M. and Walker, B. N. (2012). Usability assessment of drone technology as safety inspection tools. Electronic Journal of Information Technology in Construction, 194-212. ISSN 18744753

Kim, S. and Irizarry, J. (2015). Exploratory study on factors influencing UAS performance on highway construction projects: as the case of safety monitoring systems. Proceedings of the Autonomous and Robotic Construction of Infrastructure Conference, Ames, United States.

Melo, R. R. S., Costa, D. B., Álvares, J. S. and Irizarry, J. (2017). Applicability of unmanned aerial system (UAS) for safety inspection on construction sites. Safety science 98, 174-185. https://doi.org/10.1016/j.ssci.2017.06.008

Nakajima, K., Masuda, Shinichi., and Nakajima, S. (2017). Exploring Ways to Capture and Facilitate Work-as-Done That Interact with Health Information Technology. Resilience Health Care: Reconciling Work-as-Imagined and Work-as-Done. CRC Press, vol. 3, pp. 61-69. 
Perrow, C. (1984). Normal accidents: Living with high risk systems. Basic Books.

Polet, P., Vanderhaegen, F., Amalberti, R. (2003). Modelling border-line tolerated conditions of use (BTCU) and associated risks. Safety Science 41, 111-136. https://doi.org/10.1016/S09257535(02)00037-1

Rasmussen, J. (1997). Risk management in a dynamic society: a modelling problem. Safety Science 27, 183-213. https://doi.org/10.1016/S0925-7535(97)00052-0

Reason, J. (1990). Human error. Cambridge, Cambridge University Press.

Saurin, T. A. (2017). Removing Waste While Preserving Slack: The Lean and Complexity Perspectives. Proceedings of the $25^{\text {th }}$ Annual Conference of the International Group for Lean Construction (IGLC), Heraklion, Greece, pp. 209-216.

Saurin, T. A., Formoso, C. T., Cambraia, F. B., \& Howell, G. (2005). A cognitive system engineering perspective of construction safety. Proceedings of the $13^{\text {th }}$ Annual Conference of the International Group for Lean Construction (IGLC), Sydney, Australia, pp. 521-529.

Saurin, T. A., Rosso, C. B., and Colligan, L. (2017) Towards a Resilient and Lean Health Care. Resilience Health Care: Reconciling Work-as-Imagined and Work-as-Done. CRC Press, vol. 3, pp. 3-17.

Schafer, D., Abdelhamid, T. S., Mitropoulos, P., and Howell, G. A. (2008). Resilience engineering: a new paradigm for safety in lean construction systems. Proceedings of the $16^{\text {th }}$ Annual Conference of the International Group for Lean Construction (IGLC), Manchester, UK.

Woods, D. D. (2006). Essential characteristics of resilience. Resilience engineering: Concepts and precepts, Ashgate, pp. 21-34. 